\title{
The CFTR and EGFR relationship in airway vascular growth, and its importance in cystic fibrosis
}

\author{
Jay A. Nadel
}

Affiliation: Dept of Medicine, Cardiovascular Research Institute and Division of Pulmonary and Critical Care Medicine, University of California, San Francisco, CA, USA.

Correspondence: J.A. Nadel, UCSF School of Medicine, Box 0130, 513 Parnassus Avenue, Room S1183, San Francisco, CA 94143-0130, USA. E-mail: jay.nadelaucsf.edu

0

$@ E R S p u b l i c a t i o n s$

Describing the battle between lung defences and atmospheric invaders e.g. viruses: the winner determines our viability http://ow.ly/p7y0H

In this issue of the European Respiratory Journal, MARTIN et al. [1] focus on the life-threatening complication of haemoptysis in cystic fibrosis (CF). The authors examine the role of vascular endothelial growth factor (VEGF)-A on angiogenesis in the normal state and in CF. They report that CF airways at transplantation show increased vascularity and increased VEGF-A expression. In normal human airway epithelial cells they report that VEGF-A synthesis is produced via activation of the epidermal growth factor receptor (EGFR) and is suppressed by a selective EGFR inhibitor. Most novel and exciting are the findings that in airway epithelial cells containing cystic fibrosis transmembrane conductance regulator (CFTR), inhibition of CFTR increases EGFR activation and that an inhibitor of EGFR activation prevents the VEGF-A synthesis induced by suppression of CFTR, suggesting that there is a reciprocal relationship between CFTR and EGFR. Both CFTR and EGFR coexist on the airway epithelial surface. EGFR activation results in "pro-inflammatory responses"; in addition to VEGF-A, this includes other products such as interleukin-8 (a potent neutrophil chemoattractant) [2] and mucins [3]. The fact that inhibition of CFTR results in exaggerated production of VEGF-A suggests that CFTR normally suppresses the EGFR "inflammatory" responses.

VEGF-A production occurs in response to EGFR activation. The study by MARTIN et al. [1] found that suppression of CFTR increased EGFR activation, but only after $8 \mathrm{~h}$. This suggests that a complicated series of signalling events is involved in the activation of EGFR as a consequence of CFTR suppression. Research on the signalling pathways involved remains to be investigated.

The study by MARTIN et al. [1] describes the role of airway epithelial cell signalling in the regulation of bronchial vessel growth. Activation of EGFR promotes peribronchial angiogenesis, and the presence of CFTR on the epithelial surface inhibits this host response (the production of new bronchial vessels). The details of the cell signalling involved in the interactions of EGFR and CFTR remain unknown and may extend to a variety of actions. Furthermore, they can also provide insights into novel regulatory functions of CFTR.

CFTR gene malfunction in $\mathrm{CF}$ is a topic of great interest. In addition, recent studies suggest that changes in CFTR activity can also occur in subjects without CF. For example, DRANSFIELD et al. [4] recently reported that smokers have reduced lower airway CFTR activity compared to healthy nonsmokers. The authors suggest that acquired CFTR dysfunction may contribute to COPD pathogenesis. It should also be noted that chronic smoking results in increased expression of EGFR, which could suppress CFTR activity. Thus, the level of activity of EGFR could modulate the expression and activity of CFTR.

Received: June 242013 | Accepted: June 262013

Conflict of interest: None declared.

Copyright @ERS 2013 
Why should EGFR and CFTR coexist on the epithelial surface and play reciprocal roles in signalling of responses such as bronchial angiogenesis? The question of the potential roles of the airway epithelial surface in host defence occurred to us in 1960 when we found that cigarette smoke (an environmental pollutant) particles are deposited on the epithelial surface exerting deleterious effects on lung function [5]. The potential roles of the epithelial luminal surface in epithelial defence returned to us a decade later, when we discovered that the community pollutants sulfur dioxide [6] and ozone [7] cause airway narrowing due to smooth muscle contraction.

From consideration of these atmospheric irritants and their roles in atmospheric "invasion" and in host "defences", we concluded that the airway epithelium plays a key role in regulating responses to a variety of inhaled "irritants" and that the airway epithelial luminal surface plays a key role in alerting host defence mechanisms. Herein, I suggest that the evolution of important luminal airway epithelial signalling developed when fish migrated to land and oxygenation by the gills was improved by the development of lungs [8]. On land, inhalation of air provided a more efficient mode of oxygenation of body tissues. However, the environment also contains particulates such as viruses, bacteria, allergens and cigarette smoke, which are inhaled and deposited on airway luminal surface. It has been suggested that it is necessary for the host to detect and provide defences against the environmental invaders. The adequacy of the host defences will determine the outcome. The site of the early epithelial signalling in response to inhaled foreign particulates is logically on the luminal epithelial surface. For example, viruses cannot multiply when they are deposited on the surface of cells; penetration into the cytoplasm is necessary for multiplication. Thus, early detection and signalling is best to occur by the host before the virus enters the cell.

It is well recognised that CF is a disease caused by the CFTR gene, which results in abnormal airway epithelial ion transport [9]. The disease is characterised by infection and abnormal mucous secretion. Thus, Burgel et al. [10] have previously shown that in CF at the time of transplantation, the majority of small airways show mucous plugging in CF. However, the relationship between the abnormal CFTR gene and the pathobiology of CF lung disease is still unknown (discussed in [11]). We know that multiple abnormalities of great pathological impact exist in CF such as abnormal mucous secretion, recruitment and activation of leukocytes, and exaggerated angiogenesis [1], which are implicated in the pathology of CF. However, we do not understand exactly how the abnormal CFTR molecule leads to disease. We urgently need to learn the precise relationship between the abnormal CFTR in CF and the pathophysiology of the disease in order to prevent or treat the disease most effectively.

In considering the abnormalities that exist in CF patients and their variability among CF patients, differences in exposure to atmospheric "invaders" should be considered. Inhalation of irritants such as cigarette smoke is known to have deleterious effects on lung function, and these effects can be exaggerated in chronic airway diseases such as CF. This is the subject of a recent editorial [12]. Cigarette smoking is a notorious cause of deleterious lung function, whose documentation stretches back over many decades [5]. Thus, a variety of inhaled invaders that stimulate responses such as VEGF may also have the potential of producing exaggerated responses and, thus, in aggravating CF disease.

In summary, CF airways have increased VEGF-A, a vascular growth factor. An abnormality in the EGFR gene is responsible for CF disease, but the mechanism by which CFTR expression results in the pathological manifestation of CF disease is not clear. Here, MARTIN et al. [1] make fascinating observations that suggest that CFTR and the pro-inflammatory epithelial cell receptor EGFR have a reciprocal action on VEGF-A. The present article explores the implications of the coexistence of EGFR and CFTR on the airway epithelial surface and suggests that together these two molecules may reciprocally modulate a variety of responses, especially those related to those lung defence responses.

\section{References}

1 Martin C, Coolen N, Wu Y, et al. CFTR dysfunction induces vascular endothelial growth factor synthesis in airway epithelium. Eur Respir J 2013; 42: 1553-1562.

2 Richter A, O'Donnell RA, Powell RM, et al. Autocrine ligands for the epidermal growth factor receptor mediate interleukin-8 release from bronchial epithelial cells in response to cigarette smoke. Am J Respir Cell Mol Biol 2002; 27: 85-90.

3 Takeyama K, Dabbagh K, Lee HM, et al. Epidermal growth factor system regulates mucin production in airways. Proc Natl Acad Sci USA 1999; 96: 3081-3086.

4 Dransfield MT, Wilhelm AM, Flanagan B, et al. Acquired cystic fibrosis transmembrane conductance regulator dysfunction in the lower airways in COPD. Chest 2013; 144: 498-506.

5 Nadel JA, Comroe JH Jr. Acute effects of inhalation of cigarette smoke on airway conductance. J Appl Physiol 1961; 16: 713-716.

6 Nadel JA, Salem H, Tamplin B, et al. Mechanism of bronchoconstriction during inhalation of sulfur dioxide. J Appl Physiol 1965; 20: 164-167.

7 Goldsmith JR, Nadel JA. Experimental exposure of human subjects to ozone. J Air Pollut Control Assoc 1969; 19: 329-330. 
8 Burgel PR, Nadel JA. Plugging (publicising) to prevent mucous plugging. Eur Respir J 2010; 36: 1236-1238.

9 Cohen TS, Prince A. Cystic fibrosis: a mucosal immunodeficiency syndrome. Nat Med 2012; 18: 509-519.

10 Burgel PR, Montani D, Danel C, et al. A morphometric study of mucins and small airway plugging in cystic fibrosis. Thorax 2007; 62: 153-161.

11 Moriceau S, Lenoir G, Witko-Sarsat V. In cystic fibrosis homozygotes and heterozygotes, neutrophil apoptosis is delayed and modulated by diamide or roscovitine: evidence for an innate neutrophil disturbance. J Innate Immun 2010; 2: 260-266.

12 Annesi-Maesano I, Heinrich J, Ayres JG, et al. Why an ERJ series on air pollution? Eur Respir J 2012; 40: 12-13. 